\title{
EPITELIOPATÍA PIGMENTARIA PLACOIDE POSTERIOR MULTIFOCAL AGUDA. ESTUDIO DE 16 CASOS
}

\section{ACUTE POSTERIOR MULTIFOCAL PLACOID PIGMENT EPITHELIOPATHY. STUDY OF 16 CASES}

\author{
BURÉS-JELSTRUP A ${ }^{1}$, ADÁN A², CASAROLI-MARANO R ${ }^{2}$
}

\section{RESUMEN}

Objetivo: La Epiteliopatía Pigmentaria Placoide Posterior Multifocal Aguda (EPPPMA) es una enfermedad infrecuente que afecta a individuos jóvenes y de probable etiología inflamatoria. El objetivo del estudio es analizar las principales características clínicoepidemiológicas de esta enfermedad en una serie de pacientes.

Métodos: Se presenta un estudio retrospectivo de 16 pacientes diagnosticados de EPPPMA. Se han estudiado sus características demográficas (edad, sexo) y clínicas: evolución de la agudeza visual y del cuadro retiniano, enfermedades sistémicas asociadas y respuesta al tratamiento. Se han recogido datos angiográficos, autofluoresecencia y tomografía de coherencia óptica (OCT) en algunos pacientes.

Resultados: La edad media en nuestra serie es de 26,75 años sin preferencias por el sexo. La media de agudeza visual final en nuestra serie ha sido de 0,73 . Cuatro pacientes presentaron enfermedad sistémica asociada a la EPPPMA. 11 pacientes recibieron tratamiento con corticoesteroides (un paciente corticoesteroides e inmunosupresores) y cinco no recibieron tratamiento.

Conclusiones: En nuestra serie la edad media se encuentra por debajo de los 30 años y no hubo diferencias en cuanto al sexo, siendo estos resultados congruentes con lo descrito en la literatura.

\section{ABSTRACT}

Objective: Acute Posterior Multifocal Placoid Pigment Epitheliopathy (APMPPE) is a rare disease with a probable inflammatory component which mostly affects young patients. The aim of our study was to analyse the demographic and clinical features of this disease in a group of 16 patients.

Methods: Sixteen patients with APMPPE were included in this study. We analyzed their demographic data (age, sex) and the most relevant clinical findings: visual acuity and retinal disease outcome, association with other systemic diseases and response to treatment. We also collected data from fluorescence angiography, autofluorescence and optical coherence tomography (OCT) in some of the patients.

Results: Average age at diagnosis was 26.75 years with no sex predilection. Average final visual acuity (Snellen Scale) in our study was 0.73 . Four patients presented with a systemic disease related to the APMPPE. Eleven patients were treated with oral steroids (one patient with steroids and cytotoxic agents) while the remaining 5 patients received no treatment.

Conclusions: In our patients, the average age at diagnosis was less than 30 years, with no sex predilection, as previously described by many authors.

\footnotetext{
Recibido: 31/5/06. Aceptado: 18/4/07.

Hospital Clinic de Barcelona, Barcelona, España.

1 Licenciada en Medicina.

2 Doctor en Medicina.

Correspondencia:

A. Adán Civera

Hospital Clinic de Barcelona. Dpto. de Oftalmología

C/. Villarroel, 170

08036 Barcelona

España

E-mail: AMADAN@clinic.ub.es
} 
El pronóstico visual es bueno independientemente del tratamiento, aunque hay casos de mal pronóstico visual, especialmente aquellos con afectación foveal precoz.

Palabras clave: Epiteliopatía pigmentaria posterior pigmentaria multifocal aguda, epitelio pigmentario de la retina, coroides, angiografía fluoresceínica, angiografía con verde de indocianina, tomografía de coherencia óptica, vasculitis sistémica.
The visual outcome is usually good regardless of the treatment given, although there are cases with a bad visual outcome, especially those with foveal involvement when initially seen (Arch Soc Esp Oftalmol 2007; 82: 291-298).

Key words: acute posterior multifocal placoid pigment epitheliopathy, retinal pigment epithelium, choroid, fluorescein angiography, indocyanine green angiography, optical coherence tomography, systemic vasculitis.

\section{INTRODUCCIÓN}

Descrita por primera vez por Gass en 1968 (1), la Epiteliopatía Pigmentaria Placoide Posterior Multifocal Aguda (EPPPMA) es una enfermedad poco común, de posible etiología inflamatoria caracterizada por la aparición de varias lesiones placoides confluyentes, de color blanco-amarillento que afectan al epitelio pigmentario de la retina (EPR) y que se localizan preferentemente a nivel del polo posterior.

La enfermedad suele aparecer en individuos jóvenes, frecuentemente por debajo de los 30 años y afecta por igual a ambos sexos. Los síntomas de aparición de la EPPPMA incluyen una pérdida rápida de agudeza visual con escotoma central o paracentral. También pueden aparecer otros síntomas como miodesopsias y fotopsias.

En general se considera una enfermedad de pronóstico favorable con resolución de las lesiones en polo posterior en días o semanas y con buena recuperación de la agudeza visual aunque hay casos de peor pronóstico con un mal resultado visual final, especialmente aquellas formas con afectación foveal precoz, formas de debut a edades tardías o formas de presentación atípica (con papilitis, vasculitis retiniana, oclusiones vasculares retinianas, etc.) $(2,3)$.

La etiología de la EPPPMA es poco conocida aunque existen muchos datos a favor de una posible naturaleza inflamatoria. La transitoriedad de los síntomas y su relativamente frecuente asociación a cuadros virales prodrómicos previos (sobre todo cuadros de vías respiratorias altas) ha llevado a varios autores a considerar a la EPPPMA una enfermedad inflamatoria a nivel del EPR o coroides superficial. La hipofluorescencia precoz típica de las lesiones que aparece en la angiografía fluores- ceínica y especialmente en la angiografía con verde de indocianina (4) son sugestivos de isquemia coroidea, quizá secundaria a vasculitis coroidea como principal mecanismo de la enfermedad. Esta hipótesis vendría apoyada por las varias asociaciones que existen descritas de la EPPPMA con diversos tipos de vasculitis sistémicas como la enfermedad de Wegener (5), la sarcoidosis, vasculitis cerebrales (6) y otros estados de estimulación del sistema inmune como son las infecciones o reacciones post-vacunación.

En general la enfermedad no requiere tratamiento aunque se suelen utilizar corticoesteroides en los casos con lesiones extensas en polo posterior, casos bilaterales y casos con afectación macular. Las recurrencias son raras pero si se presentan suelen indicar un peor pronóstico.

\section{SUJETOS, MATERIAL Y MÉTODOS}

Presentamos un estudio retrospectivo en el que se han incluido 16 pacientes (26 ojos afectados) diagnosticados de EPPPMA.

El objetivo de este estudio consiste en estudiar las principales características epidemiológicas (edad, sexo) de estos pacientes así como las características clínicas: agudeza visual y su evolución durante el tiempo de seguimiento, tasa de bilateralidad, número de brotes en el tiempo de seguimiento, las posibles asociaciones con enfermedades sistémicas (como ya se ha comentado en el apartado anterior) y respuesta al tratamiento. Asimismo también se valoraron los hallazgos angiográficos y de la tomografía de coherencia óptica (OCT) en aquellos pacientes a los que se les realizaron exploraciones complementarias. 


\section{RESULTADOS}

Según los resultados obtenidos en el seguimiento de esta serie de 16 pacientes se evidencia que la edad al diagnóstico se sitúa por debajo de los 30 años de edad. La edad media en nuestra serie fue de 26,75 años, presentando el paciente más joven 13 años en el momento del diagnóstico y el mayor, 44 años.

No encontramos diferencias en cuanto a la distribución por sexos, siendo ocho de los 16 pacientes varones (50\% de los pacientes) y los ocho restantes mujeres (50\% de los pacientes). En cuanto a la distribución por edad en función del sexo, las diferencias tampoco fueron muy significativas aunque se apreció una cierta tendencia a un debut más precoz de la enfermedad entre las mujeres, las cuales presentaban una media de edad de 24,65 años respecto a la media de edad al diagnóstico de 28,87 años en los varones.

En cuanto a la bilateralidad de la enfermedad, nueve pacientes presentaron afectación sólo en un ojo mientras que los siete pacientes restantes presentaron alteraciones en los dos ojos. De estos siete pacientes con afectación bilateral, cinco presentaron afectación bilateral desde el momento del diagnóstico (figs. 1-4) y 2 empezaron con un brote unilateral que al poco tiempo se bilateralizó.

La media de brotes clínicamente bien diferenciados en el tiempo que presentaron durante su evolución los pacientes fue de 1,44. La mayoría de pacientes (12 de 16 pacientes) presentaron un sólo

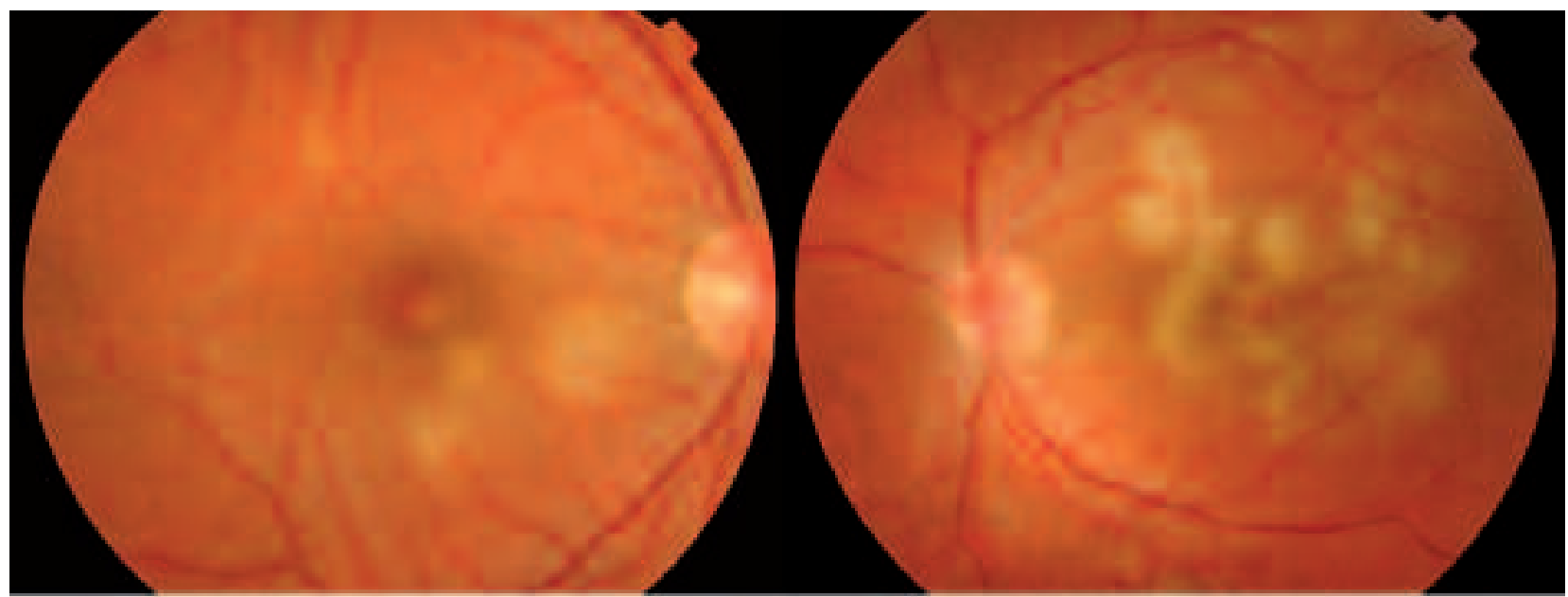

Figs. 1 a 4: Caso 7: Imágenes arriba: afectación bilateral simultánea de ambos ojos en fase activa. Imágenes de abajo: imágenes de autofluorescencia en las que se aprecia la hipoautofluorescencia del EPR en las zonas de inflamación activa. 
Tabla I

\begin{tabular}{llccc}
\hline & AV inicio brote & \multicolumn{2}{c}{ AV final } \\
\hline & OD & OI & OD & OI \\
\hline Caso 1 & $\mathbf{0 , 3}$ & 1,0 & $\mathbf{0 , 8}$ & $\mathbf{0 , 3}$ \\
Caso 2 & $\mathbf{0 , 1}$ & 1,0 & $\mathbf{0 , 8}$ & 1,0 \\
Caso 3 & $\mathbf{0 , 4}$ & 0,9 & $\mathbf{1 , 0}$ & 1,0 \\
Caso 4 & 0,9 & $\mathbf{0 , 2}$ & 0,9 & $\mathbf{0 , 2}$ \\
Caso 5 & $\mathbf{0 , 7}$ & $\mathbf{0 , 6}$ & $\mathbf{1 , 0}$ & $\mathbf{0 , 4}$ \\
Caso 6 & $\mathbf{0 , 6}$ & 0,8 & $\mathbf{0 , 5}$ & 1,0 \\
Caso 7 & $\mathbf{0 , 6}$ & $\mathbf{0 , 3}$ & $\mathbf{0 , 5}$ & $\mathbf{0 , 3}$ \\
Caso 8 & $\mathbf{1 , 0}$ & 1,0 & $\mathbf{1 , 0}$ & 1,0 \\
Caso 9 & $\mathbf{0 , 1}$ & 1,0 & $\mathbf{0 , 1}$ & 0,8 \\
Caso 10 & 1,0 & $\mathbf{0 , 0 5}$ & 1,0 & $\mathbf{1 , 0}$ \\
Caso 11 & $\mathbf{0 , 6}$ & 1,0 & $\mathbf{0 , 5}$ & 1,0 \\
Caso 12 & $\mathbf{0 , 2}$ & $\mathbf{0 , 1}$ & $\mathbf{0 , 9}$ & $\mathbf{0 , 8}$ \\
Caso 13 & $\mathbf{1 , 0}$ & $\mathbf{1 , 0}$ & $\mathbf{0 , 6}$ & $\mathbf{0 , 6}$ \\
Caso 14 & 1,0 & $\mathbf{0 , 0 5}$ & $\mathbf{1 , 2}$ & $\mathbf{0 , 9}$ \\
Caso 15 & $\mathbf{0 , 5}$ & $\mathbf{0 , 6}$ & $\mathbf{1 , 0}$ & $\mathbf{1 , 2}$ \\
Caso 16 & $\mathbf{0 , 5}$ & 1,0 & $\mathbf{1 , 0}$ & 1,0 \\
\hline \hline
\end{tabular}

En la tabla se muestran las agudezas visuales (AV) en OD y OI de los 16 pacientes en el momento inicial del primer brote y la $\mathrm{AV}$ al final del seguimiento. En negrita se marcan las AV de aquellos ojos afectados y en letra normal aquellos ojos que no presentaron ningún brote durante el seguimiento. Algunos casos unilaterales al inicio de la enfermedad se bilateralizaron con el tiempo (casos 1 y 14), mientras que fueron casos bilaterales desde el inicio 5 casos (casos $5,7,12,13$ y 15) y unilaterales solamente, 9 pacientes (casos 2, 3, 4, 6, 8, 9, 10, 11 y 16).

brote, mientras que dos pacientes presentaron dos brotes y dos pacientes presentaron tres y cuatro brotes respectivamente durante el tiempo de seguimiento.

El tiempo entre brotes de aquellos pacientes que presentaron más de un brote de la enfermedad fue variable, oscilando desde las 2 semanas a los 5 años entre brotes.

En cuanto a la agudeza visual (AV) de los pacientes, se resume en la tabla I los resultados de AV al inicio del brote y los resultados al final del seguimiento.

La duración total del seguimiento fue muy variable, con un rango que oscilaba entre los 1,5 meses a los 6 años. Se consideró $A V$ final aquella AV que presentaba el paciente cuando su cuadro retiniano se hallaba completamente estabilizado, es decir, lesiones en polo posterior pigmentadas de aspecto cicatricial y con ningún signo de actividad. Se observó una tendencia a pérdidas de agudeza visual en general leves y con una buena recuperación, siendo la media de AV en la Escala de Snellen en el momento del brote activo de 0,45 y de 0,73 al final del seguimiento, una vez inactiva la enfermedad. Así, en el momento del diagnóstico, un 47,61\% de los pacientes presentaba una AV menor a 0,5 mientras que el $52,38 \%$ restante tenía una AV igual o mayor de 0,5. Al final del seguimiento, un 78,26\% de los pacientes había recuperado visión de 0,5 o mayor mientras que un 21,73\% mantenían AV por debajo de 0,5 .

De los 16 pacientes, 12 no presentaron ninguna enfermedad sistémica que pudiera considerarse asociada a su enfermedad ocular, antes ni después del diagnóstico de EPPPMA. Tres pacientes presentaron un cuadro viral inespecífico con fiebre y malestar general semanas previas al brote de EPPPMA, cursando uno de ellos con aumento de la velocidad de sedimentación y eritema nudoso en extremidad inferior. Otro caso fue diagnosticado de vasculitis sistémica con c-ANCA positivo a raíz del diagnóstico de EPPPMA, pero la biopsia de mucosa nasal fue negativa para enfermedad de Wegener. Este caso en concreto fue el de más difícil control terapéutico ya que presentó cuatro brotes muy seguidos con progresión rápida de la enfermedad que sólo se pudo controlar con tratamiento inmunosupresor (Prednisona, Metotrexate y Ciclofosfamida en bolus), aunque el resultado final de AV fue bueno, siendo de 1,2 y 0,9 en OD y OI respectivamente (figs. 5 a 10).

En cuanto al tratamiento que recibieron estos pacientes, cinco pacientes no recibieron ningún tratamiento, nueve pacientes recibieron tratamiento sólo con corticoesteroides por vía oral (en general con pautas de $1 \mathrm{mg} / \mathrm{kg} /$ día en pauta descendiente), un paciente recibió Prednisona via oral más una inyección subtenoniana de Triamcinolona y otro paciente requirió tratamiento múltiple con inmunosupresores (Prednisona, Metotrexate y bolus de Ciclofosfamida) para el control de la enfermedad.

De aquellos ojos que recibieron tratamiento, la media de AV final fue de 0,68 , mientras que en los que no recibieron tratamiento fue de 0,81 .

Se realizó angiografía fluoresceínica (AGF) a 12 de los 16 pacientes durante la fase activa de su enfermedad. Todos los pacientes mostraron en mayor o menor grado el patrón típico de la AGF en la EPPPMA, con hipofluorescencia de las lesiones en tiempos precoces debido al efecto pantalla producido, probablemente, por las células del epitelio pigmentario de la retina (EPR) inflamadas y por la hipoperfusión coroidea. En tiempos tardíos de la AGF aparece un patrón de hiperfluorescencia difuso de las lesiones. En la fase inactiva de la enfermedad los hallazgos angiográficos ya no son tan característicos y muestran una hiperfluorescencia difusa sin pérdida de contraste debido al efecto ventana producido por la atrofia del EPR en la zona de las lesiones. 

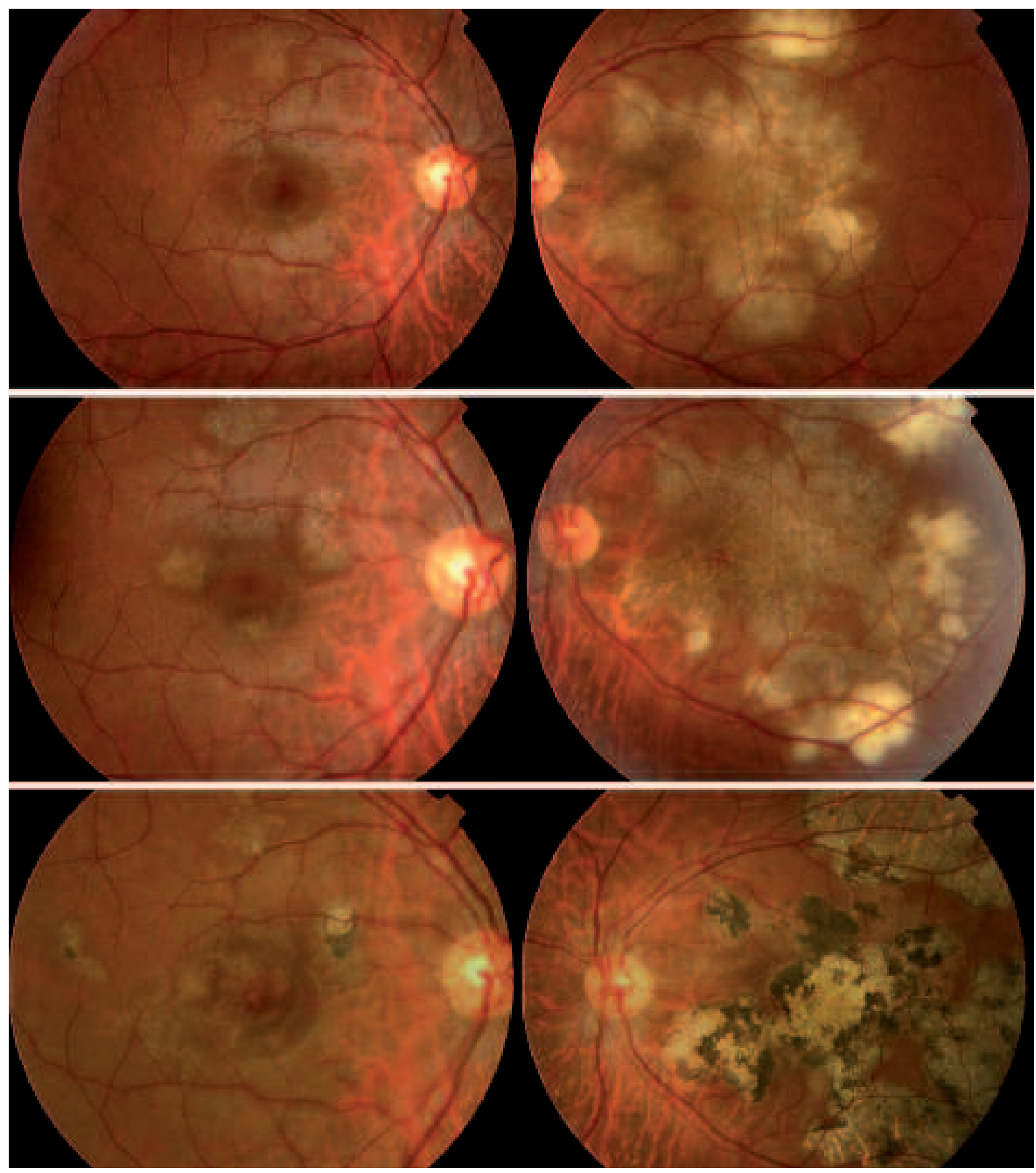

Figs. 5 a 10: Caso 14: Imágenes arriba: aspecto del fondo de ojo en OD y OI respectivamente durante el primer brote de la enfermedad. Imágenes del medio: aspecto del fondo de ojo al cabo de 20 días. Se aprecian claramente en OI la aparición de lesiones en distintas etapas evolutivas, algo característico de la EPPPMA; mientras las primeras lesiones en aparecer empiezan a pigmentarse y a presentar un aspecto cicatricial empiezan a aparecer lesiones activas en los bordes periféricos de las lesiones antiguas. Imágenes de abajo: aspecto fundoscópico una vez estabilizado el cuadro en ambos ojos. Las lesiones ya están totalmente cicatrizadas y la AV del paciente es de 1,2 y 0,9 en OD y OI respectivamente. 

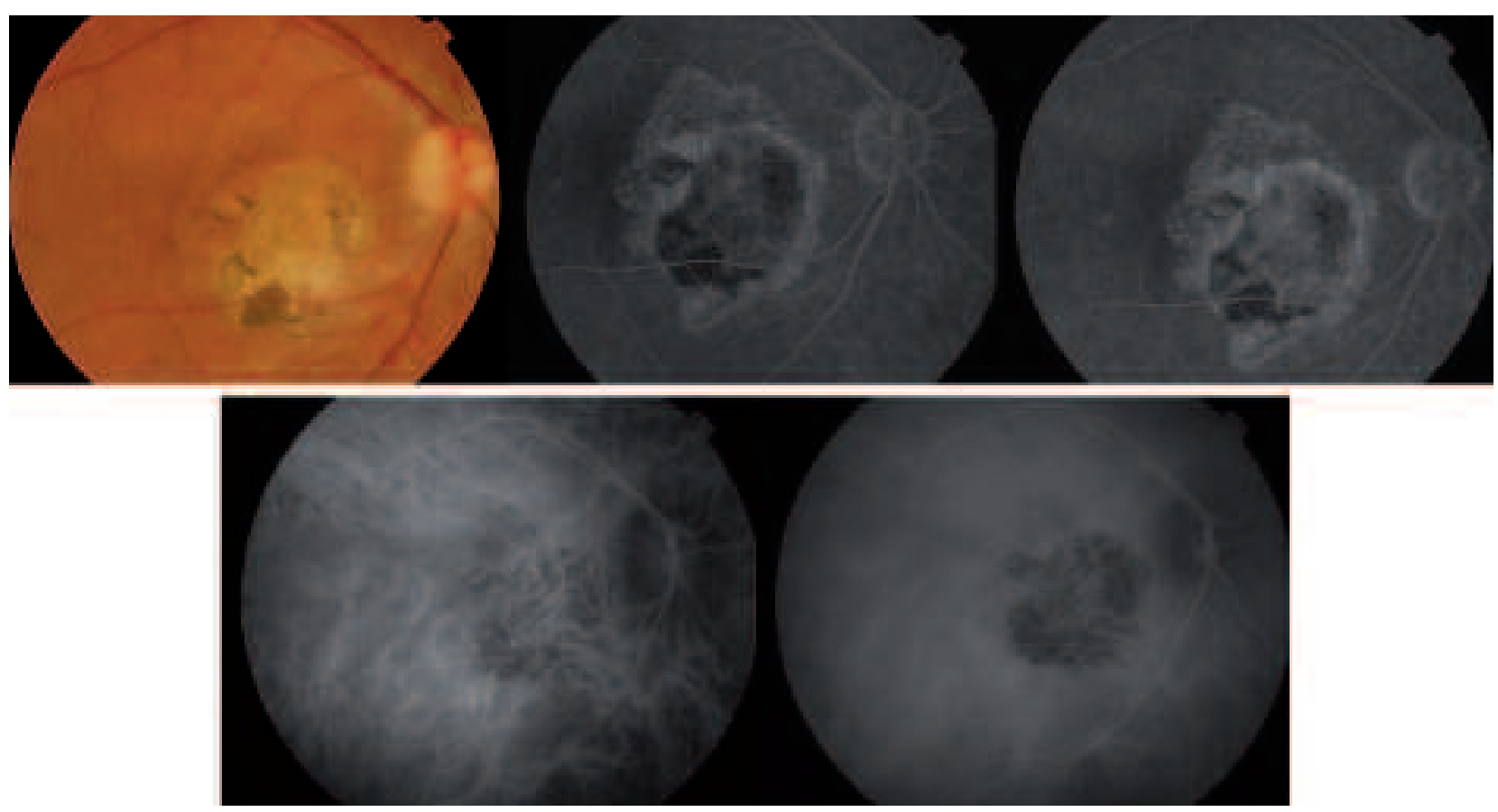

Figs. 11 a 15: Caso 6: Imágenes arriba de izquierda a derecha: aspecto fundoscópico de OD (único ojo afectado) en el que se aprecian las lesiones placoides confluyentes en polo posterior en fase previa a la cicatrización definitiva. Las siguientes dos imágenes muestran la angiografía fluoresceínica en tiempos precoces y tardios respectivamente. En tiempos precoces se aprecia una característica hipofluorescencia mientras que en tiempos tardios las lesiones muestran tendencia a la hiperfluorescencia. Imágenes de abajo de izquierda a derecha: angiografía con verde de indocianina en tiempos precoces y tardios respectivamente. Se muestra la característica hipoperfusión coroidea a nivel de las lesiones. La AV final en ese ojo fue de 0,5.

A un paciente se le realizó angiografía con verde de indocianina (figs. 11 a 15) en la que se aprecia la característica hipoperfusión coroidea a nivel lesional.

Se realizó tomografía de coherencia óptica (OCT) a cuatro de los 16 pacientes. Está descrito en la literatura la presencia de una hiperreflectividad de las capas externas de la retina sin aumento del grosor retiniano a nivel de las placas activas(7), aunque en nuestro caso no objetivamos diferencias significativas entre el aspecto tomográfico de la retina sana versus retina afectada.

\section{DISCUSIÓN}

Con los datos obtenidos se puede confirmar la tendencia descrita en la literatura sobre el debut a edades tempranas de la EPPPMA. Según nuestros datos, la edad media de presentación fue de 26,75 años, dato común en la mayoría de revisiones (8). Del mismo modo tampoco observamos una predilección de la EPPPMA por el sexo.
Existe la aceptación generalizada de que la EPPPMA es una enfermedad de afectación ocular bilateral en la mayoría de los casos. En cambio, en esta serie más de la mitad de los casos $(56,25 \%)$ presentaron afectación de un solo ojo. Este dato podría deberse a un tiempo de seguimiento corto de alguno de los pacientes, ya que la afectación del ojo contralateral puede producirse meses e incluso años después del primer brote. Aun así, lo más frecuente es que la bilateralización, si no es inmediata, se produzca al cabo de poco tiempo, en general semanas, con lo cual podríamos pensar que quizá la tasa de afectación unilateral no es tan infrecuente como previamente descrito en la literatura, al menos a la vista de nuestros resultados.

En la serie se observa también una tendencia a una buena recuperación de la $\mathrm{AV}$, en algunos casos con reestablecimiento de la AV pre-brote. Este pronóstico visual favorable se considera característico de la EPPPMA aunque hay que tener en cuenta que algunos de estos pacientes no recuperan una buena AV. En nuestros pacientes se ha observado que el 
principal factor pronóstico para predecir un mal resultado visual final fue la afectación foveolar desde el momento del diagnóstico.

Como ya se ha comentado previamente, existen casos de asociaciones sistémicas con la EPPPMA. De los 16 casos estudiados sólo tenemos constancia evidente de tres pacientes que presentaron un cuadro viral inespecífico previo al diagnóstico y un paciente que fue diagnosticado de vasculitis sistémica no filiada.

Recibieron tratamiento antiinflamatorio 11 pacientes (nueve pacientes recibieron corticoesteroides orales, un paciente Triamcinolona subtenoniana + corticoesteroides orales y un paciente recibió corticoides e inmunosupresores) mientras que cinco pacientes no recibieron tratamiento. Según los resultados observados en nuestra serie tuvieron mejores AV aquellos pacientes que no recibieron tratamiento (AV media de 0,81 frente a una AV media de 0,68 en aquellos pacientes que sí recibieron tratamiento). Aunque estos resultados pueden parecer contradictorios a primera vista, es posible que fueran los casos de peor pronóstico inicial (por ejemplo casos con afectación foveolar) los que recibieron tratamiento esteroideo, estando así el resultado visual final más influenciado por la afectación foveolar inicial que por el tratamiento recibido. Aun así, varios autores recomiendan administrar tratamiento con corticoesteroides debido a la probable naturaleza inflamatoria de la enfermedad, especialmente en aquellos casos con mala agudeza visual inicial, formas con lesiones muy extensas y las formas de presentación atípicas (9).

\section{BIBLIOGRAFÍA}

1. Gass JD. Acute posterior multifocal placoid pigment epitheliopathy. Arch Ophthalmol 1968; 80: 177-185.

2. Abu El-Asrar AM, Aljazairy AH. Acute posterior multifocal placoid pigment epitheliopathy with retinal vasculitis and papilitis. Eye 2002; 16: 642-644.

3. Allee SD, Marks SJ. Acute posterior multifocal placoid pigment epitheliopathy with bilateral central retinal vein occlusion. Am J Ophthalmol 1998; 126: 309-312.

4. Howe LJ, Woon H, Graham EM, Fitzke F, Bhandari A, Marshall J. Choroidal hypoperfusion in acute posterior multifocal placoid pigment epitheliopathy. An indocyanine green angiography study. Ophthalmology 1995; 102: 790-798.

5. Chiquet C, Lumbroso L, Denis P, Papo T, Durieu I, Lehoang $P$. Acute posterior multifocal placoid pigment epitheliopathy associated with Wegener's granulomatosis. Retina 1999; 19: 309-313.

6. O'Halloran HS, Berger JR, Lee WB, Robertson DM, Giovannini JA, Krohel GB, et al. Acute multifocal placoid pigment epitheliopathy and central nervous system involvement: nine new cases and a review of the literature. Ophthalmology 2001; 108: 861-868.

7. Souka AA, Hillenkamp J, Gora F, Gabel VP, Framme C. Correlation between optical coherence tomography and autofluorescence in acute posterior multifocal placoid pigment epitheliopathy. Graefes Arch Clin Exp Ophthalmol 2006; 244: 1219-1223.

8. Quillen DA, Davis JB, Gottlieb JL, Blodi BA, Callanan $D G$, Chang TS, et al. The white dot syndromes. Am J Ophthalmol 2004; 137: 538-550.

9. Celis Sánchez J, González del Valle F, Alonso Martínez I, Romero Royo C, López Ferrando N, Zarco Tejada JM. Desprendimientos retinianos serosos bilaterales en la epiteliopatía pigmentaria placoide multifocal posterior aguda. Arch Soc Esp Oftalmol 2001; 76: 679-682. 